\title{
Aspectos estruturais do aerossol atmosférico da Bacia Amazônica
}

\author{
Paulo Artaxo Neto (1); Celso Q. Orsini ('); Luiz Carlos Bouéres ( ${ }^{2}$ ) e Alistair Leslie ( ${ }^{2}$ )
}

\begin{abstract}
Resumo
Este trabalho apresenta um desenvolvimento dos resultados já divulgados em dois artigos anteriores, sobre o aerossol atmosférico natural de regiões da Bacia Amazônica e sobre a estrutura da matéria particulada emitida pelas queimadas que com elevada freqüência ocorrem naquelas regiões. Esses resultados foram cbtidos através de experiências realizadas em agosto-setembro de 1980, na Estações "Duque" e "Torre", para os aerossóis naturais, e em pontos da Rodovia ManausPorto Velho, até $\sim 800 \mathrm{~km}$ ao sudoeste de Manaus, para aerossóis de queimadas. (As estações Duque e Torre são instalações do INPA a, respectivamente $\sim 20$ e $80 \mathrm{~km}$ ao norte de Manaus]. Foram determinadas as concentrações dos elementos-traços $\mathrm{Al}, \mathrm{Si}, \mathrm{P}, \mathrm{S}, \mathrm{Cl}, \mathrm{K}$, $\mathrm{Ca}, \mathrm{Ti}, \mathrm{V}, \mathrm{Mn}, \mathrm{Fe}, \mathrm{Cu}, \mathrm{Br}$ e $\mathrm{Pb}$ e algumas propriedades da estrutura destes aerossóis; realizou-se também estudos de identificação das fontes desses elementos-tracos. Os novos resultados aqui apresentados referem-se às distribuições de concentrações pelo tamanho de elementos-traço dos aerossóis investigados; tazendó uso de curvas log-probabilidade e de uma nova técnica aqui introduzida, foi possível derivar curvas de concentraçăo total correspondentes aos elementos com número atômico $Z \geq 13$. $O$ uso conveniente dessas curvas permite ampla caracterização dos aerossóis em consideração.
\end{abstract}

\section{INTRODUÇÃO E METODOLOGIA}

Este trabalho apresenta resultados complementares a outros já divulgados em artigos anteriores (Orsini et al., 1981) (Artaxo et al., 1981), obtidos a partir de experiências realizadas na Amazônia simultaneamente e em paralelo ao "Projeto Queimadas 1980" do NCAR (National Center for Atmospheric Rescarch). Os resultados dessas experiências levaram à concepção de um padrão estrutural consistente do aerossol atmosférico natural da Bacia
Amazônica, e também das principais propriedades do aerossol de queimadas que freqüentemente assolam aquela região.

As amostragens de aerossóis remotos (ou naturais) foram feitas na "Estação Duque" e na "Torre", facilidades implantadas pelo INPA em meio à selva, ao norte de Manaus, distantes 20 e $80 \mathrm{~km}$, respectivamente. As medidas em queimadas foram feitas ao longo da rodovia Manaus-Porto Velho, em várias localidades, à distâncias de até $800 \mathrm{~km}$ ao Sudoeste de Manaus.

A coleta do material particulado foi feita através de impactadores em cascata de 6 estágios, que separam o aerossol em 6 faixas de diânetro aerodinâmico: 4 ụm para o estágio $5,2 \mu \mathrm{m}$ para o estágio $4,1 \mu \mathrm{m}$ para o estágio $3,0,5 \mu \mathrm{m}$ para o estágio $2,0,25 \mu \mathrm{m}$ para o estágio 1, e um filtro Nuclepore coleta a matéria particulada menor que $0,25 \mu \mathrm{m}$. Também foi utilizado o amostrador de fino e grosso, onde através de filtração sequencial separa-se o particulado grosso (Dp $>2,5 \mu \mathrm{m})$ do particulado fino $(\mathrm{Dp}<2,5 \mu \mathrm{m})$.

A análise elementar do aerossol foi feita através do método PIXE (Particle Induced $X$-Ray Emission), para os elementos com $Z \geq 13$, e do método PESA (Proion Elastic Scattering Analysis) para a medida das concentrações de Carbono, Nitrogênio e Oxigênio. As irradiações foram feitas no Instituto de Física da USP e na Florida State University. As descrições detalhadas das metodologias adotadas e das experiências realizadas estão apresentadas nas referências (Orsini et al., 1981) e (Artaxo et al., 1981).

(1) - Instituto de Física da Universidade de São Paulo, C. Postal 20.516 - CEP. 05508, São Paulo, SP, Brasil. (2) - The Florida State University, Tallahasse, Florida, 32306, USA. 


\section{RESULtados}

Nos trabalhos anteriores supra-citados apontaram-se vários resultados e conclusões das experiências em consideração; resumimos adiante os mais relevantes aos propósitos deste trabalho:

1) Foram determinadas as curvas de concentração em função do diâmetro aerodinâmico, para os seguintes elementos: $\mathrm{Al}, \mathrm{Si}, \mathrm{P}, \mathrm{S}, \mathrm{Cl}, \mathrm{K}, \mathrm{Ca}, \mathrm{Ti}, \mathrm{V}, \mathrm{Mn}, \mathrm{Fe}$, $\mathrm{Cu}, \mathrm{Zn}, \mathrm{Br}$ e $\mathrm{Pb}$. A figura 1 sintetiza esses resultados para os aerossóis naturais examinados, na forma de curvas de distribuições médias dos impactadores medidos na Estação Duque e curvas de distribuições da Torre (resultados de difícil obtenção em virtude das condições adversas de operação do equipamento de amostragem no local). A figura 2 apresenta curvas típicas dos aerossóis de regiões de queimadas. Essas curvas revelam padrões estruturais desses aerossóis relativamente aos elementos-traços; nota-se claramente a predominância do $\mathrm{S}$ e $\mathrm{K}$ para particulados finos $(<2,5 \mu \mathrm{m})$, e do $\mathrm{Si}, \mathrm{Al}, \mathrm{Ca}$ e $\mathrm{Fe}$ para particulados grossos $(>2,5 \mu \mathrm{m})$ em regiões remotas.

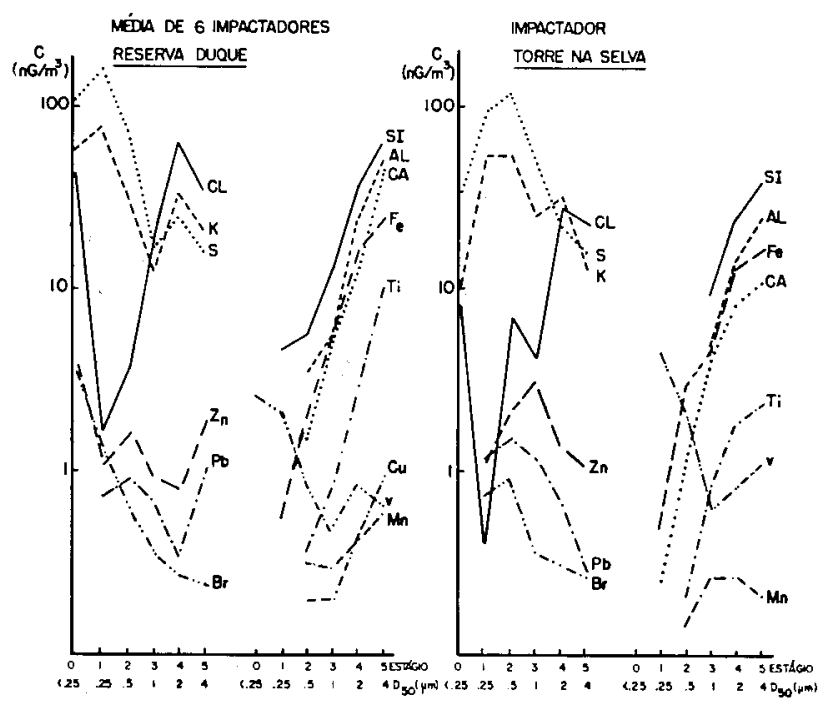

Fig. 1 - Curvas de distribuição elementar pelo tamanho da Estação Duque (média) e da Torre.

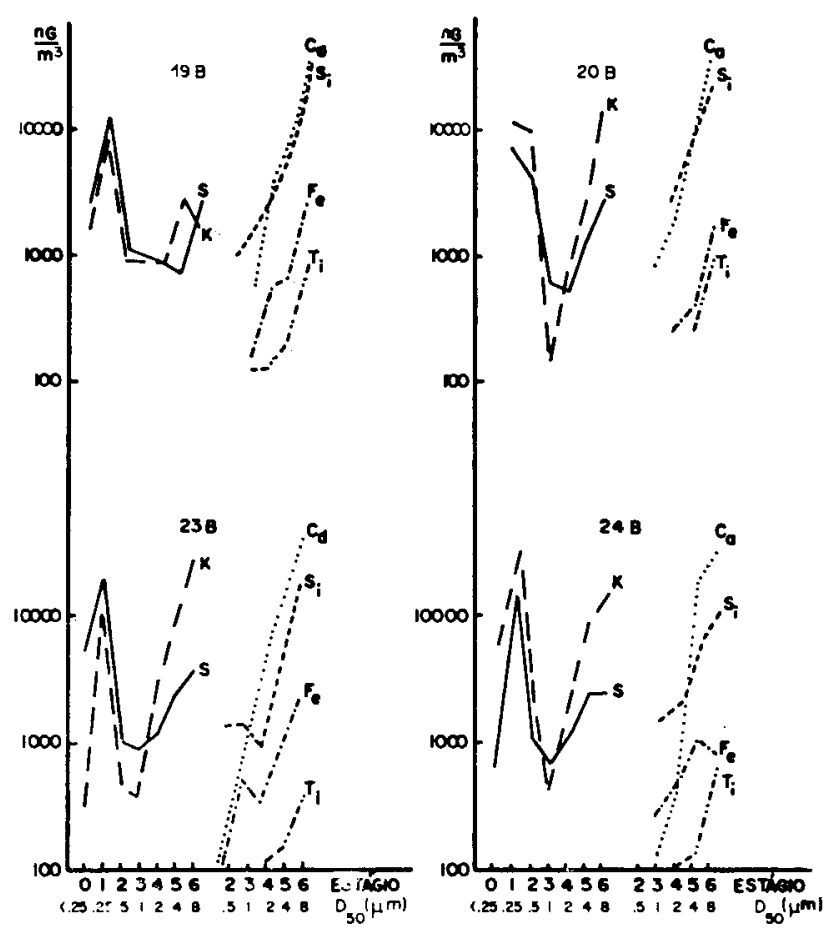

Fig. 2 - Curvas típicas de distribuição elementar pelo tamanho dos particulados de quelmadas.

2) Na Tabela 1 são apresentadas as concentrações totais dos elementos-traços encontrados nos aerossóis da Estação Duque e da Torre. Na última coluna da tabela colocou-se os valores do Fator de Enriquecimento, definido como:

$$
F E=\frac{\left(\frac{\mathrm{C}_{\mathrm{x}}}{\mathrm{CFe}_{\mathrm{Fe}}}\right)}{\left(\frac{\mathrm{C}_{\mathrm{x}}}{\mathrm{C}_{\mathrm{Fe}} \text { Torre }}\right)}
$$

onde: $\mathrm{C}_{\mathrm{X}}=$ concentração do elemento-traço $\mathrm{x}$ e $\mathrm{C}_{\mathrm{F}_{\mathrm{e}}}=$ concentração do Ferro.

$\mathrm{O}$ valor médio de $\mathrm{FE}$ resultou igual a 1,40 , fato que indica a ocorrência de contribuições antropogênicas sensíveis na Estação Duque em relação à Torre e também de uma maior contribuição de elementos do solo.

3) A tabela 2 coloca as concentrações totais dos elementos-traços em queima- 
TABELA 1 - Concentrações totais dos elementos-traços na Estação Duque (média dos valores" e na Torre, medidas por meio de impactadores em cascata (em $\mathrm{NG} / \mathrm{M}^{3}$ ), e fatores de enriquecimento.

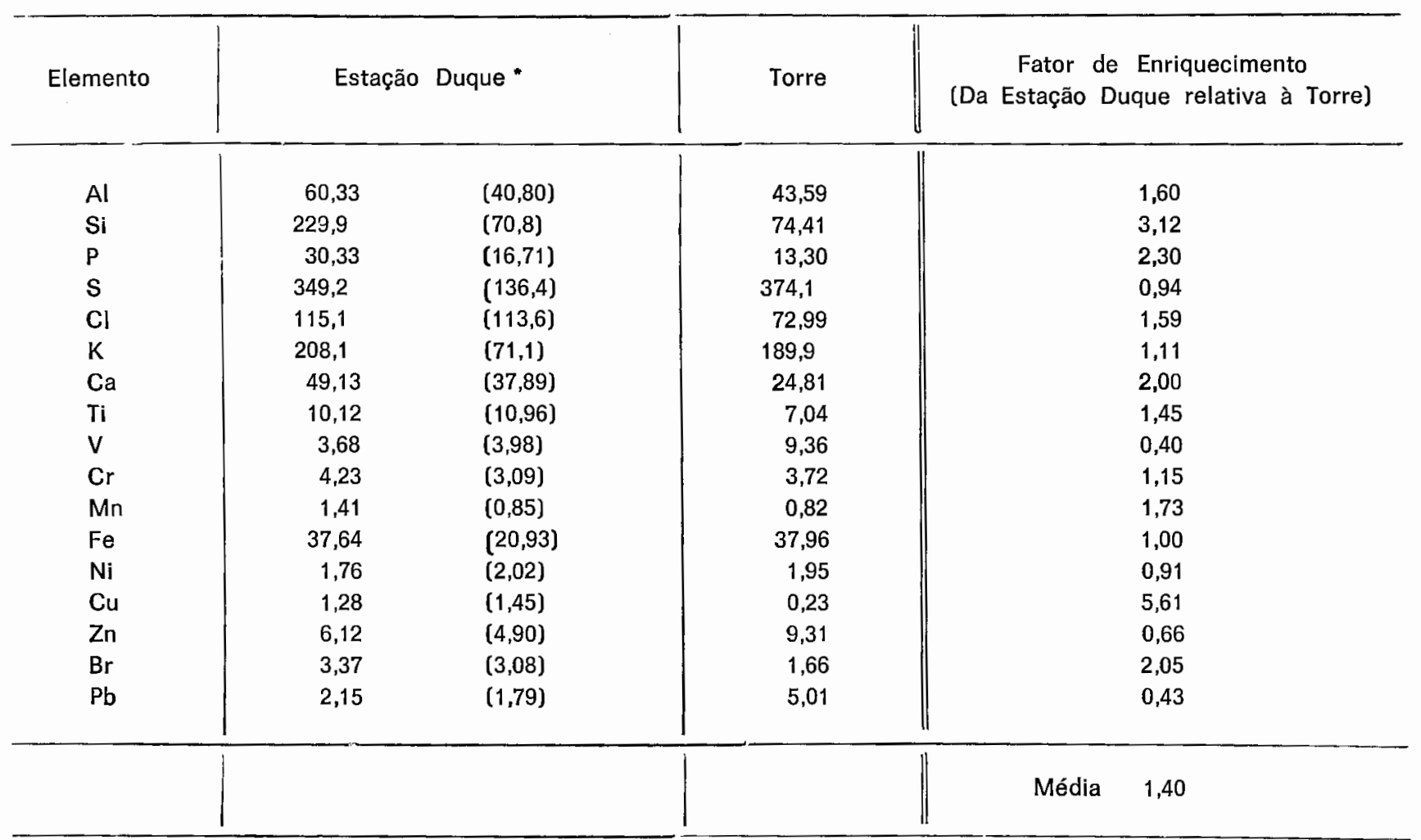

(*) - Os números entre parêntesis são os desvios padrões das distribuições dos valores usados nos cálculos das concentrações.

TABELA 2 - Concentrações totais dos elementos-traços em queimadas medidas por impactadores em cascata (EM NG $/ M^{3}$ ).

\begin{tabular}{|c|c|c|c|c|c|c|c|}
\hline Elemento & $19 \mathrm{~B}$ & $20 B$ & 21B & $22 \mathrm{~B}$ & $23 B$ & $24 \mathrm{~B}$ & $28 \mathrm{~B}$ \\
\hline $\mathrm{Al}$ & 5386 & 6264 & 2227 & 0 & 0 & 0 & 3678 \\
\hline Si & 53014 & 31812 & 9950 & 11900 & 28363 & 21850 & 43819 \\
\hline$P$ & 19723 & 20172 & 11690 & 24667 & 26173 & 32504 & 6474 \\
\hline$S$ & 17179 & 18254 & 11018 & 21890 & 20731 & 25046 & 11268 \\
\hline $\mathrm{Cl}$ & 9138 & 9170 & 6328 & 11906 & 13565 & 22232 & 8799 \\
\hline $\mathrm{K}$ & 21896 & 42378 & 25225 & 54444 & 67591 & 75091 & 15969 \\
\hline $\mathrm{Ca}$ & 45949 & 37345 & 5900 & 13193 & 68789 & 59301 & 4293 \\
\hline$T i$ & 1309 & 1234 & 304 & 347 & 797 & 1307 & 2053 \\
\hline V & 175 & 2056 & 0 & 209 & 286 & 55 & 48 \\
\hline $\mathrm{Cr}$ & 188 & 143 & 134 & 0 & 14 & 84 & 492 \\
\hline Mn & 2390 & 2405 & 475 & 1074 & 2065 & 3472 & 101 \\
\hline $\mathrm{Fe}$ & 3916 & 2816 & 2329 & 633 & 4822 & 2370 & 2725 \\
\hline $\mathrm{Ni}$ & 0 & 0 & 230 & 290 & 211 & 0 & 0 \\
\hline $\mathrm{Cu}$ & 552 & 434 & 90 & 115 & 811 & 0 & 20 \\
\hline $\mathrm{Zn}$ & 701 & 318 & 187 & 489 & 181 & 201 & 94 \\
\hline $\mathrm{Br}$ & 359 & 727 & 0 & 0 & 20 & 9 & 147 \\
\hline $\mathrm{Pb}$ & 277 & 1482 & 203 & 237 & 543 & 662 & 48 \\
\hline $\mathrm{Sr}$ & 760 & 786 & 87 & 268 & 946 & 956 & 56 \\
\hline
\end{tabular}


das. Esses valores são cerca de duas ordens de grandeza superiores aos naturais, e revelam predominância de $\mathbf{S i}$, $\mathrm{S}, \mathrm{K}$ e $\mathrm{Ca}$ no aerossol.

4) As porcentagens, em massa dos elementos leves $\mathrm{C}, \mathrm{N}$ e $\mathrm{O}$ em relação aos elementos com $Z \geq 13$, medida nos aerossóis da Duque, Torre e em Queimadas foram as seguintes:

\begin{tabular}{c|c|c|c|c|c}
\hline & $\mathrm{C}$ & $\mathrm{N}$ & $\mathrm{O}$ & $\begin{array}{c}\text { Elementos } \\
\text { com }\end{array}$ & $\mathrm{C}+\mathrm{N}+\mathrm{O}$ \\
$\mathrm{Z} \geq 13$ & \\
\hline Estação Duque & 60 & 6,6 & 25 & 8,4 & 92 \\
Torre & 55 & 6,2 & 17 & 7,6 & 78 \\
Queimadas & 78 & 1,4 & 20 & 0,7 & 99 \\
\hline
\end{tabular}

Como se vê, a participação do $\mathrm{C}, \mathrm{N}$ e $\mathrm{O}$ vai desde $78 \%$ para o aerossol natural da Torre, para chegar a $99 \%$ em regiões de queimadas, fato que mostra a estrutura diferenciada dos aerossóis de queimadas; nos aerossóis urbanos esta participação é em torno de $75 \%$.

5) Definindo o Fator de Enriquecimento do aerossol em relação à biomassa pela relação:

$$
F E=\frac{\left(\frac{C_{x}}{C_{r e f} \text { aerossol }}\right.}{\left(\frac{C_{x}}{C_{\text {ref biomassa }}}\right)}
$$

onde: $\mathrm{C}_{\mathrm{x}}=$ concentração do elemento $\mathrm{x}, \mathrm{C}_{\mathrm{ref}}=$ concentração do elemento de referência, tomando como elemento de referência o enxofre, para a moda fina do particulado (Dp $<2,5 \mu \mathrm{m})$ tem-se:

\begin{tabular}{crl} 
Elemento & \multicolumn{1}{c}{ F. } & . \\
$\mathrm{Si}$ & 1,2 & $\pm 0,9$ \\
$\mathrm{Ti}$ & 9 & \pm 5 \\
$\mathrm{Fe}$ & 0,5 & $\pm 0,2$ \\
$\mathrm{Ca}$ & 0,004 & $\pm 0,003$ \\
$\mathrm{P}$ & 0,07 & $\pm 0,07$ \\
$\mathrm{Cl}$ & 0,2 & $\pm 0,2$ \\
$\mathrm{~K}$ & 0,12 & $\pm 0,10$
\end{tabular}

Observamos um forte "empobrecimento" do $\mathrm{Ca}$ e do $\mathrm{P}$, sugerindo que esses elementos não tomam parte no mecanismo de exsudação dos vegetais; 0 "enriquecimento" do Ti mostra que este elemento deve ser originado por outra fonte (neste caso, o solo).

6) Para a componente grossa do aerossol natural, tomando-se como elemento de referência o Silício, e como fonte o solo, tem-se:

$\begin{array}{cc}\text { Elemento } & F . E . \\ \mathrm{Cl} & 3 \times 10^{3} \pm 1 \times 10^{3} \\ \mathrm{~S} & 2 \times 10^{2} \pm 1 \times 10^{2} \\ \mathrm{P} & 1 \times 10^{2} \pm 1 \times 10^{2} \\ \mathrm{Ca} & 10 \pm 5 \\ \mathrm{Ti} & 5 \pm 3 \\ \mathrm{Fe} & 3 \pm 1\end{array}$

Sobressai-se neste quadro o violento enriquecimento dos elementos $\mathrm{Cl}, \mathrm{S}$ e $P$, evidenciando a participação da biomassa na sua produção.

7) Para os aerossóis de queimadas na moda fina, tomando como base o Enxofre, obtivemos:

$\begin{array}{cc}\text { Elemento } & F . \\ \mathrm{K} & 0,5 \pm 0,5 \\ \mathrm{P} & 0,9 \pm 0,9 \\ \mathrm{Cl} & 2 \pm 1 \\ \mathrm{Si} & 4 \pm 5 \\ \mathrm{Ti} & 4 \times 10^{1} \pm 3 \times 10^{\prime} \\ \mathrm{Ca} & 0,01 \pm 0,01\end{array}$

Estes dados, apesar das variâncias altas, decorrentes das dificuldades experimentais, sugerem a biomassa como geradora dos elementos $\mathrm{K}, \mathrm{P}$ e $\mathrm{Cl}$ nos aerossóis de queimadas.

8) Uma análise estatística das curvas de distribuição, revelou que no aerossol natural existe alta correlação $(>0.92)$ entre os elementos $\mathrm{C}, \mathrm{N}$ e $\mathrm{O}$, bem como desses elementos com $\mathrm{Si}, \mathrm{P}, \mathrm{S}, \mathrm{Cl}$, $\mathrm{K}, \mathrm{Ca}, \mathrm{Ti}$ e $\mathrm{Fe}$, fato indicativo de que esses elementos leves ( $\mathrm{C}, \mathrm{N}$ e $\mathrm{O}$ ) são os principais constituintes do substra- 
to dos particulados do ar. Na componente grossa do mesmo aerossol nátural, observa-se alta correlação entre 03 elementos $\mathrm{Si}, \mathrm{Cl}, \mathrm{Ca}, \mathrm{Ti}$ e $\mathrm{Fe}$, indicando que estes elementos compõe o mesmo particulado. Nos aerossóis de queimadas, existe uma correlação forte entre $\mathrm{C}, \mathrm{N}$ e $\mathrm{O}$, e valores menores nas correlações entre estes elementos e os elementos-traços, exceptando-se o $\mathrm{S}$ e o K. Na componente grossa, os elementos do solo $\mathrm{Si}, \mathrm{Ca}, \mathrm{Ti}$ e $\mathrm{Fe}$ intercorrelacionam-se bem, e ainda persiste a correlação entre $\mathrm{S}$ e $\mathrm{K}$.

A estes resultados, já registrados e exaustivamente discutidos em artigos anteriores, acrescentamos agora os produtos de uma nova via de análise utilizada na caracterização de aerossóis atmosféricos.

\section{Distribuição de MASSA EM AEROSSÓIS ATMOSFÉRICOS SEMI-ESTACIONÁRIOS}

É fato conhecido, embora as razões para isso não estejam ainda totalmente claras, que o material particulado, quando em equilíbrio, comparece nos aerossóis atmosféricos, de tal modo que sua massa, por unidade de volume de ar distribui-se Log-Normalmente pelo tamanho das partículas. Dessa maneira, a curva de distribuição definida por $\mathrm{dm} / \mathrm{d} \log \mathrm{Dp} / \mathrm{unidade}$ de volume de ar versus $\log \mathrm{Dp}$, onde $\mathrm{dm}$ representa a diferencial de massa do particulado com diâmetro aerodinâmico ent:e $\mathrm{Dp} e$ $\mathrm{Dp}+\mathrm{dDp}$, tem a forma da distribuição log-Normal, isto é:

$\frac{\mathrm{dm}}{\mathrm{d} \log \mathrm{Dp}}=\frac{1}{\sqrt{2 m \log \sigma}} \exp -\left(\frac{(\log \mathrm{Dp}-\log \bar{D})^{2}}{2 \log ^{2} \sigma}\right)$

onde $\overline{\mathrm{D}}=$ diâmetro aerodinâmico de massa média, $\sigma=$ desvio padrão geométrico da distribuição.

Graficando-se esta função em papel monolog obtem-se uma gaussiana e em papel log-probabilidade, uma reta.

Os aerossóis atmosféricos em equilíbrio, apresentam em sua faixa semi-estacionária
$(0,1 \mu m \leq D p \leq 30 \mu m)$ duas distribuiçõcs log-normais distintas: a primeira correspondente ao particulado fino, constitui a moda de acumulação ou moda fina, e centraliza-se em torno de $\sim 0,4 \mu \mathrm{m}$; a segunda, que se refere à moda grossa tem um pico em cerca de $7 \mu \mathrm{m}$ (Suck et al., 1977). A moda fina ( $D p \gtrsim 2,5 \mu \mathrm{m})$ e a moda grossa ( $D p \gtrless 2,5 \mathrm{~m}$ ) são essencialmente independentes quanto às suas origens, transformações e processos de remoção (Whitby, 1978). Constitui interessante exemplo deste comportamento, o aerossol natural da Amazônia, investigado na Reserva Duque e na Torre, cuja moda fina é constituída essencialmente de elementos provenientes da floresta, como $\mathrm{S}$ e $\mathrm{K}$, através do processo de exsudação das folhas (Lawson, 1978) enquanto que a moda grossa contém, basicamente, elementos do solo, como Al, $\mathrm{Si}, \mathrm{Ca}, \mathrm{Ti}, \mathrm{Fe}$ e outros, gerados pela ação do vento.

\section{Parâmetros obtidos PARA o AEROSSOL} ATMOSFÉRICO DA AMAZÔNIA

A distribuição de tamanho para $Z \geqslant 13$ pode ser simulada a partir de dados de impactador em cascata analisados por PIXE e do modelo Log-Normal, bastando, para tanto, obterse os valores de 3 parâmetros, para cada moda (fina e grossa), que determinam univocamente toda a distribuição:

$\overline{\mathrm{D}}$ : diâmetro aerodinâmico de massa média (ou pico da distribuição de tamanho);

$\sigma$ : desvio padrão geométrico da distribuição (ou largura a meia altura do pico);

C : constante de normalização.

A constante de normalização é necessária para ajustar a amplitude das curvas Log-Normais aos valores de concentrações das distribuições de tamanho medidas.

Os objetivos deste tratamento são dois: 1..) parametrizar as curvas de distribuição de tamanho, obtendo os valores de $\bar{D}$ e $\sigma$ a partir dos resultados do impactador em cascata; 2.) fazer uma simulação global da curva de concentração versus diâmetro da partí- 
cula para os elementos com $Z \leq 13$ e para $0.1 \leq \mathrm{Dp} \leq 30 \mu \mathrm{m}$. Para tanto, colocamos as concentrações elementares da figura $1 \mathrm{em}$ um gráfico acumulativo em papel log-probabilidade. Se a distribuição é log-normal, tem-se uma reta para cada uma das modas fina e grossa. Este gráfico está na figura 3 para os dados da Torre e na figura 4 para as concentrações elementares medidas na Estação Duque. Deste gráfico, obtem-se o valor de $\overline{\mathrm{D}}$, que corresponde ao diâmetro aerodinâmico para $50 \%$ da concentração total medida, e o valor da largura $\sigma$ tomando a razão do diâmetro correspondente à $84,1 \%$ em relação ao de $50,0 \%$ (Seinfeld, 1975). Os resultados médios para cada moda são:

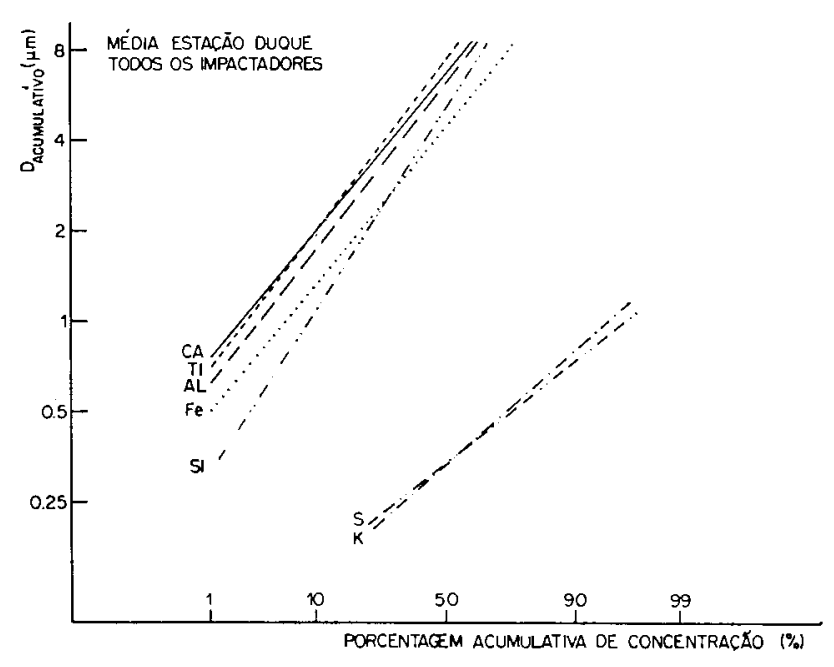

Fig. 4 - Gráfico Log-probabilidade para o aerossol da Estação Duque.

\begin{tabular}{l|lccc}
\hline \multirow{2}{*}{ Torre } & Moda fina & (S e K) & Moda grossa & (Fe, Ti, Si, Ca e Al) \\
\cline { 2 - 5 } & $\overline{\mathrm{D}}=0,54 \mu \mathrm{m}$ & $\sigma=1,8 \mu \mathrm{m}$ & $\overrightarrow{\mathrm{D}}=3,9 \mu \mathrm{m}$ & $\sigma=2,1 \mu \mathrm{m}$ \\
\hline Duque & $\overline{\mathrm{D}}=0,34 \mu \mathrm{m}$ & $\sigma=1,9 \mu \mathrm{m}$ & $\overline{\mathrm{D}}=5,9 \mu \mathrm{m}$ & $\sigma=2,8 \mu \mathrm{m}$ \\
\hline
\end{tabular}

Ve-se claramente, que na Duque o valor de $\bar{D}$ na moda grossa é maior que na Torre, devido à presença de clareiras e estradas a cerca de $2 \mathrm{~km}$ do local da amostragem, enquanto que na Torre o solo é coberto por vegetação e árvores altas por vários quilômetros do local da amostragem. A amplitude da distri-

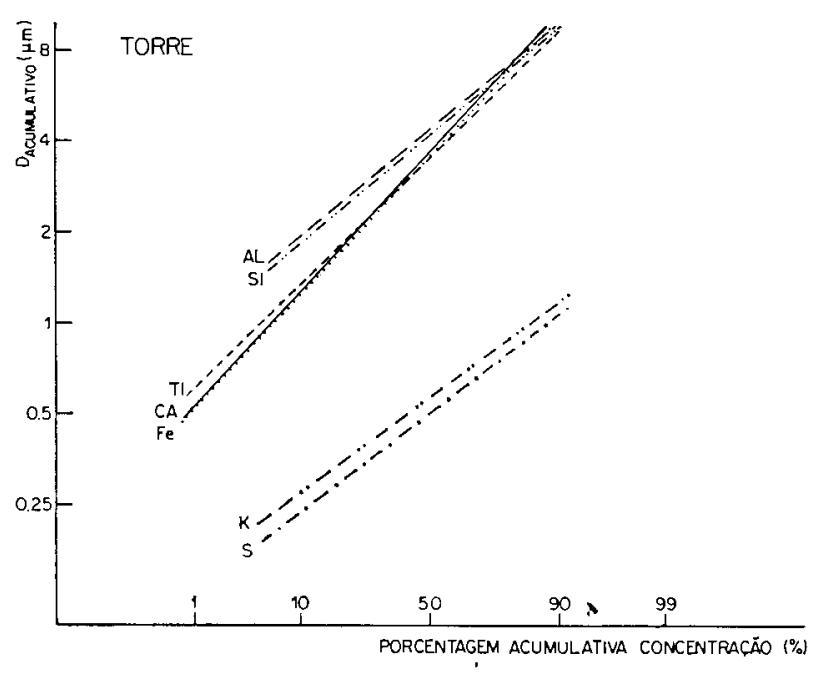

Fig. 3 - Gráfico Log-probabilidade para o aerossol da Torre. buição na moda grossa é maior na Reserva Duque pela mesma razão.

Para avaliar a constante de normalização, fez-se uma simulação das curvas de distribuição com estes valores de $\bar{D}$ e $\sigma$; obtidos do gráfico Log-probabilidade, e ajustou-se $C_{F}$ e $\mathrm{C}_{G}$ (respectivamente, constantes de normalização da moda fina e grossa), de modo que a curva resultante represente a soma das distribuições elementares para $Z \leq 13$. Desta maneira, obteve-se:

\begin{tabular}{|c|c|c|}
\hline & Moda fina & Moda grossa \\
\hline Torre & $C_{F}=307$ & $C_{G}=282$ \\
\hline Duque & $C_{F}=429$ & $\mathrm{C}_{\mathrm{G}}=707$ \\
\hline
\end{tabular}

A partir destes parâmetros simulou-se a curva de concentração em função de Dp para $Z \leq 13$ colocada na figura 5 . Estima-se uma variância de $10 \%$ para os valores de $\bar{D}$ e $\sigma$, e de $30 \%$ para os valores de $C_{F}$ e $C_{G}$. 


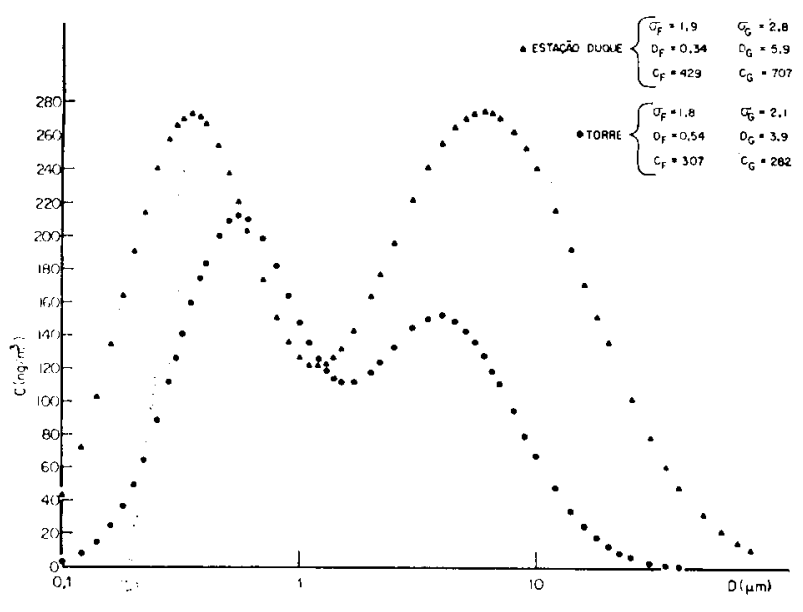

Fig. 5 - Distribuição de tamanho para $Z \geq 13$ na Torre e na Estação Duque.

\section{CONCLUSões}

Ve-se na figura 5 que a separação entre as moda fina e grossa ocorre para $D p \sim 1,5 \mu \mathrm{m}$, sendo que para a Torre a moda fina é proeminente em relação à moda grossa, enquanto que na reserva Duque, as duas modas possuem amplitudes próximas.

Por outro lado, os resultados das retas log-probabilidade confirma as análises estatísticas feitas sobre os dados, mostrando (tanto para a Duque como para a Torre) que $\mathrm{S}$ e $\mathrm{K}$ são altamente correlacionados e participam do mesmo particulado, devido aos valores semelhantes obtidos para $\bar{D}$ e $\sigma$ de cada elemento. Para a moda grossa, os elementos $\mathrm{Al}, \mathrm{Si}, \mathrm{Ti}$, $\mathrm{Ca}$ e Fe forneceram valores de $\overline{\mathrm{D}}$ e $\sigma$ com va riância de no máximo $10 \%$, também confirmando os resultados das análises estatística e de fatores de enriquecimento.

Por fim, salienta-se que neste artigo fol introduzida uma nova metodologia que permitiu determinar a curva global de distribuição de um aerossol e que forneceu resultados concordantes com os métodos usuais de análise de dados de aerossóis atmosféricos.

As medidas das concentrações na Torre estão entre as mais baixas já obtidas no mundo, com resultados comparáveis aos aerossóis remotos de Chacaltaya na Bolívia e em outros lugares longínquos (Winchester, 1981).

Para os aerossóis remotos, a participação de $\mathrm{C}, \mathrm{N}$ e $\mathrm{O}$ é de cerca de $80 \%$ da massa total, enquanto que para de queimadas esta porcentagem sobe a $99 \%$, evidenciando a diferenciação entre a estrutura destes dois tipos de aerossóis.

\section{Agradecimentos}

Os autores agradecem a toda a equipe do INPA, particularmente ao Dr. Ozório J. M. Fonseca, vice-diretor em exercício por ocasião das experiências, e à Dra. Maria de Nazaré Góes Ribeiro, que forneceu todo o apoio à realização das amostragens na Torre e na Estação Duque.

A experiência teve apoio financeiro do CNPq, e da National Science Foundation dos E.U.A.

\section{SUMMARY}

The results presented on this paper may be considered as complementary to the ones published a two previous papers about the natural atmospheric aerosol of the Amazon Basin, and the effects, on these physicalchemical systems of the large scale brushfires carried out from time to time on that region. The experiments have been performed in August-September, 1980, simultaneously to the ones of the "Projeto Queimadas 1980" promoted by the National Center for Atmospheric Research from the U.S.A. The new results here in presented are size distribution concentration data as logprobability curves for the detected trace-elements from these curves, by introducing a new technique, we were able to derived the corresponding log-normal curves. These last curves can be used conveniently to characterize the atmospheric aerosol system which is under investigation.

\section{REFERENCIAS BIBLIOGRAFICAS}

ARTAXO NETTO, P.; ORSINI, C.Q.; TABACNIKS, M.H.; BOUÉRES, L.C.; LESLIE, A.

1981 - Características dos aerossóis atmosférico natural e de queimadas da Bacia Amazônica. Anais da Academìa Brasileira de Ciênclas (em publicação). 
LAWSON, D.R.

1978 - Chemistry of the Natural Aerosol: a case study in South America Ph.D. Thesis.

ORSINI, C.Q.; NETTO, P.A.; TABACNIKS, M.H.

1981 - Preliminary data on atmospheric aerosol of the Amazon Basin. Atmospheric Environment. (em publicação).

SEINFELD, J.H.

1975 - Air Pollution: Physical and Chemical Fundamentals McGraw-Hill Book Company, N. York.
SUCK, S.H.; MIDDLETON, P.B.; BROCK, J.R.

1977 - On the Multimodality of Density Functions of Pollutant Aerosols. Atmospheric Environment, 11: 251-255.

WHITBY, K.T.

1978 - The Physical Characteristics of Sulfur Aerosols. Atmospheric Environment, 12: 135-159.

WINCHESTER, J.W.

1981 - Particulate Matter and Sulfur in the Natural Atmosphere. Nuclear Instruments and Methods, 181: 367-381.

(Aceito para publicação em 13/04/82) 